\title{
Lysogenic Mycobacteria: Phage Variations and Changes in Host Cells
}

\author{
By EDITH MANKIEWICZ, MAIMO LIIVAK \\ AND SABINE DERNUET \\ Royal Edward Chest Hospital, Montreal, Canada
}

(Accepted for publication 8 November 1968)

\begin{abstract}
SUMMARY
Lysogenic conversion of mycobacteria by phage Roy results in reciprocal changes in the phase and in the host cells. The temperate phage released after lysogenization differs from the converting phage in its host range. The lysogenic derivatives show marked changes in their colony morphology and in their sensitivity to phagolysis. Some, but not all, of the lysogenic bacteria have suffered losses in enzymic activity. An attempt is made to distinguish between host-determined and phage-determined variations.
\end{abstract}

\section{INTRODUCTION}

Phage-determined changes in colony morphology, enzymic activity and other properties of lysogenized mycobacteria were described in 196I (Mankiewicz, 196I). White, Foster \& Lyon (1962) reported alterations of colony morphology in strain ATCC 607 after lysogenization with phages $\mathrm{R}_{\mathrm{I}}$ and $\mathrm{D}_{32}$. Russell, Jann \& Froman (1963), however, did not detect differences between stock cultures of strain ATCC607 and derivatives of this strain produced by lysogenization with one or other of five phages. Vandra \& Takats (1965) lysogenized Mycobacterium fribergensis and $M$. rabinowitsch with lytic phages. The staining properties, colony morphology, amidase and catalase activity and synthesis of niacin were similar in both the phage-sensitive parent strains and their lysogenic derivatives. Juhasz \& Boenicke (I966) reported 'changes' in colony morphology, and decrease in the growth rate of $M$. phlei $\mathrm{F} 89$ after its lysogenization with phage B2. Recently, Jones \& White (1968) described the conversion of colony morphology, nitrate reductase activity and Tween hydrolysis of strain ATCC 607 associated with its lysogenization by phages D 29 and B4.

Changes in bacterial properties obtained with some phage-host systems but not with others could be due to the selective effect of the phage on mycobacterial mutants that pre-existed within the particular bacterial population.

The following experiments were undertaken to demonstrate that, within some pure and homogeneous mycobacterial strains, lysogenization determines host- and phagevariants, while in other phage-sensitive strains no such reciprocal changes occur. This indicates that lysogenization may involve, or determine, different genetic processes in different mycobacterial strains. 


\section{METHODS}

Mycobacteriophage Roy was isolated in our laboratories from the sputum of a patient with thoracic sarcoidosis. Repeated cultures of sputum from this patient had been negative for mycobacteria. Since its isolation in liquid medium enriched with strain ATCC607 (R) this phage had been propagated on this strain only, and its host range and lytic activity have remained the same. Phage Roy produces $0.8-\mathrm{I} \cdot 0 \mathrm{~mm}$. clear plaques with regular borders on strains ATCC607 and on TMCI5I5. At a phage: bacterium ratio of $\mathrm{I}: \mathrm{IO}$, its lytic cycle in ATCC 607 consists of an absorption period of $30 \mathrm{~min}$. (65\% absorption), a latent period of $70 \mathrm{~min}$., a rise period of $30 \mathrm{~min}$. The lytic cycle is completed in approximately $2 \frac{1}{2} \mathrm{hr}$. The average burst size is 92 . Electronmicroscopic studies show this phage to be formed by a head hexagonal in structure measuring 92-94 $\mathrm{m} \mu$ in length and 53-54 $\mathrm{m} \mu$ in width. The length of the tail is approximately $250 \mathrm{~m} \mu$.

Media for phage study. The RVB liquid medium for the preparation of phage filtrates and the RVA agar medium for phage typing and phage titrations (in r.t.d. and p.f.u.) were prepared and used according to the recommendations of Redmond \& Ward (1966).

Table I. Mycobacterial strains used in this study

\begin{tabular}{|c|c|}
\hline Species and group & Strain or source \\
\hline $\begin{array}{l}\text { M. tuberculosis, var. hominis } \\
\text { M. tuberculosis, var. bovis }\end{array}$ & $\begin{array}{l}\text { H } 37 \text { RV, Steenken, Trudeau } \\
\text { BCG, Université de Montréal }\end{array}$ \\
\hline \multicolumn{2}{|l|}{ Atypical Mycobacteria: } \\
\hline \multirow[t]{3}{*}{ Runyon group I } & TMC I 201 \\
\hline & TMC I 203 \\
\hline & $\begin{array}{l}\text { TMC 1204 } \\
\text { I518, Royal Edward Chest Hospital }\end{array}$ \\
\hline \multirow[t]{2}{*}{ Runyon group II } & TMC1302 \\
\hline & $\begin{array}{l}\text { TMCI306 } \\
\text { I } 8685 \text {, Royal Edward Chest Hospital }\end{array}$ \\
\hline \multirow[t]{2}{*}{ Runyon group III } & TMC I 403 \\
\hline & TMC I404 \\
\hline M. fortuitum & TMC I5I2 \\
\hline M. smegmatis & ATCC 607 \\
\hline M. fribergensis & $\begin{array}{l}\text { TMC I515 } \\
\text { E. Vandra, Budapest }\end{array}$ \\
\hline M. phlei & E. Vandra, Budapest \\
\hline M. rabinowitsch & E. Vandra, Budapest \\
\hline M. stercoides & E. Vandra, Budapest \\
\hline M. lacticola & ATCC 9626 \\
\hline Mycobacterium sp. & ATCC9033 \\
\hline Mycobacterium sp. & $\operatorname{ATCC} 4243$ \\
\hline
\end{tabular}

Bacteria. The twenty-one mycobacterial strains included in this study are tabulated in Table I. Before use, they were plated on RVA agar medium and one colony was selected, suspended in nutrient broth (Difco) and replated on solid medium. This process of colony selection was repeated twice for each strain. Three colonies were selected from the last plating and propagated separately. The three subcultures of each strain were submitted to the tests indicated below, in order to compare their properties and to ascertain the homogeneity and purity of each strain. 
The following characters were studied: growth rate in liquid and on solid medium at $37^{\circ}$ and at $22^{\circ}$; colony morphology and the conditions for their pigmentation; cord formation and acid fastness in liquid medium; utilization of paraffin as the sole carbon source in Czapek's mineral medium (without sucrose); catalase and peroxydase activity (Tirunaranay \& Fischer, 1957), the amidase reactions (Boenicke, 1958), synthesis of niacin (Konno, 1957) and, for bacilli from group IV, the transformation of ammonium ferrocitrate (Tison, Tacquet \& Devulder, 1964). Sensitivity to streptomycin, isoniazid and to p-aminosalicylic acid was determined as well as the sensitivity of each strain to phagolysis by phages DS6, G4E, AG i of Redmond's phage-typing scheme (Redmond, 1963). The presence or absence of an inducible prophage was studied in filtrates of cultures that had been irradiated with u.v. for $30 \mathrm{sec}$. at $10 \mathrm{~cm}$. distance ( 15 watt G.E. germicidal tube) or had been exposed to hydrogen peroxide (I0\%) or Actinomycin-C (10 $\mathrm{gg}$./ml.). Lysis of the mycobacterial strains by phage Roy was determined in the following way: the bacteria were plated on solid medium and incubated for three hours. Droplets of a phage suspension that just produced an area of clear lysis on ATCC 607 were deposited on the bacterial lawns. The plates were re-incubated and read after intervals of $48 \mathrm{hr}$ to Io days. Only complete clearing of the 'spots' was considered as true lysis.

Lysogenization of the mycobacterial strains. Five cultures of each bacterial strain in RVB medium were infected with phage Roy in a phage/cell ratio of $5: I$. The infection took place during the exponential growth period of the mycobacteria: the fast growing bacteria were infected after three hr incubation; the slow growing organisms, after I $8 \mathrm{hr}$. The cultures were re-incubated for $3 \mathrm{hr}$ and one ml. samples of the cultures were added to equal volumes of phage antiserum that neutralized $98 \%$ of free phage particles. After three $\mathrm{hr}$ incubation with the antiserum, the cultures were centrifuged and the sediments were suspended, each in $0.2 \mathrm{ml}$. of RVB medium. These suspensions were then plated on RVA agar. Colonies produced by bacteria that survived the phage infection were isolated and propagated in RVB medium to which Tween 80 at a concentration of $0.1 \%$ was added in order to avoid re-infection by free phage particles. Subcultures on RVA medium and on Loewenstein medium were then submitted to a study of their bacterial properties by means of the above-mentioned tests.

As proposed by Russell, Jann \& Froman (1963), temperate phages released by lysogenic mycobacteria are designated by the name of the phage, followed in parentheses by the name of the mycobacterium in which lysogeny was established; lysogenic derivatives are designated by the name of the parent strain, followed in parentheses by the name of the converting phage.

\section{RESULTS}

Phage variations. Phage Roy (607) and phage Roy (1515) produce clear spots on mycobacterial strains 607 and I5I5. Under experimental conditions for lysogenization, they also produce phage-resistant lysogenic bacteria within these two strains. These release phage particles of the same lytic pattern and produce the lysogenic conversion of the same bacterial strains as did the virulent phage. By its lytic activity, phage Roy is a phage specific for Mycobacterium smegmatis.

The nineteen remaining strains resisted phagolysis. Seven strains, however, produced lysogenic colonies after incubation with phage Roy. The number of colonies under- 
going lysis after u.v.-induction of the phage-infected population varied from 65 to $90 \%$. The phage particles released by the lysogenic bacteria showed marked changes in lytic pattern. With one exception, the lytic spectrum had expanded and included, in every instance, the respective host strain. The strains susceptible to lysogenization by temperate phages were the same that were susceptible to the parent phage; efficiency to establish the lysogenic state was, however, much greater for the temperate phages. The lytic spectrum of phage Roy and of the phage variants is summarized in Table 2.

Table 2. Lytic activity of phage Roy and its host-determinant variants

\begin{tabular}{|c|c|c|c|c|c|c|c|c|}
\hline Indicator & $\begin{array}{l}\text { Phage } \\
\text { Roy } \\
(607)\end{array}$ & (1518) & (1302) & (1306) & (1512) & (Phlei) & $\begin{array}{l}\text { (Ster- } \\
\text { coides) }\end{array}$ & $\begin{array}{c}\text { (Lacti- } \\
\text { cola) }\end{array}$ \\
\hline Strain 607 & + & + & + & + & + & - & + & + \\
\hline 1515 & + & + & + & + & + & - & + & + \\
\hline 1518 & - & + & + & + & - & - & - & - \\
\hline 1302 & - & - & + & + & - & - & - & - \\
\hline 1306 & - & - & + & + & - & - & - & - \\
\hline I512 & - & - & - & - & + & - & - & - \\
\hline M. phlei & - & - & - & - & - & + & + & - \\
\hline M. stercoides & - & - & - & - & + & + & + & + \\
\hline M. lacticola & - & - & - & - & - & - & - & + \\
\hline
\end{tabular}

Table 3. Changes in growth rate, colony morphology, pigmentation of colonies and in the microscopic aspect in mycobacteria lysogenized with phage Roy

\begin{tabular}{|c|c|c|c|c|}
\hline $\begin{array}{l}\text { Lysogenic } \\
\text { derivative of }\end{array}$ & $\begin{array}{l}\text { Growth } \\
\text { rate }\end{array}$ & Colony & Pigmentation & Bacterial morphology \\
\hline 607 & Delay & R. S nippled & Earlier & $\begin{array}{l}\text { Swollen cytoplasma which } \\
\text { lost acid fastness. Heavy } \\
\text { beading }\end{array}$ \\
\hline 1515 & Delay & $R, S$ to $S$ & No change & Partial loss of acid fastness \\
\hline 1518 & Delay & $\mathbf{S}$ & No change & Partial loss of acid fastness \\
\hline 1302 & Delay & $\mathbf{R}, \mathbf{S}$ & $\begin{array}{l}\text { Dissociation into pig- } \\
\text { mented and non- } \\
\text { pigmented umbilicated } \\
\text { colonies }\end{array}$ & Partial loss of acid fastness \\
\hline 1306 & Delay & $\mathbf{R}$ to $\mathbf{S}$ & No change & No change \\
\hline 1512 & Delay & $\mathbf{S}$ & No change & Partial loss of acid fastness \\
\hline M. phlei & Delay & $\mathbf{S}$ & No change & $\begin{array}{l}\text { Relative loss of acid fast- } \\
\text { ness }\end{array}$ \\
\hline M. stercoides & Delay & $\begin{array}{l}\text { S, nippled } \\
\text { colonies }\end{array}$ & No change & $\begin{array}{l}\text { Heavy beading, partial loss } \\
\text { of acid fastness }\end{array}$ \\
\hline M. lacticola & Delay & $\mathbf{S}$ & $\begin{array}{l}\text { Increase in pigmen- } \\
\text { tation }\end{array}$ & $\begin{array}{l}\text { Heavy beading, partial loss } \\
\text { of acid fastness }\end{array}$ \\
\hline & & $\mathbf{R}=\mathbf{R o u}$ & $\mathrm{S}=$ Smooth & \\
\hline
\end{tabular}

Changes in bacterial properties associated with lysogenization. Ten mycobacterial variants were isolated from the nine strains lysogenized by phage Roy: two from strain 1302; one from each of the following: 607, 1515, 1518, 1306, 1512, Mycobacterium phlei, $M$. stercoides and $M$. lacticola. The lysogenic derivatives shared the ability to produce phage particles, the lytic activity of which has been described previously. They all showed decrease in their growth rate during the first two or three 
subcultures. Instead of the rough or wrinkled colonies of most of the parent strains, lysogenic strains produced smooth colonies, some of them heaped or nippled. Microscopically, bacteria from these colonies showed a relative loss in acid fastness that affected mainly the cytoplasma; Much's granula were purple and caused heavy 'beading' of the bacteria. The lysogenic derivatives were resistant to phagolysis by the converting phage and by the phage variants. The colonies of 607 (Roy) and 1515

Table 4. Comparison between strain ATCC607 and strain ATCC607 (Roy) isolated 8 days after phage infection

Character on test

Colony morphology on

\section{RVA agar}

Pigmentation

Bacterial morphology and acid fastness

Growth rate in RVB
medium
Paraffin utilization
Cord formation
Amidase reactions:
Urease
Pyrazinamidase
Nicotinamidase
Acetamidase
Benzamidase
Succinamidase
Niacin synthesis
Catalase activity
Peroxydase
Nitrate reduction
Transformation of
ammonium ferrocitrate
Sensitivity to:
Streptomycin
(IO-IOO mcg./ml.)
Isoniazid
(I-IO mcg./ml.)
PAS (I-IOO mcg./ml.)
Lysis by phage:
DS 6 A
G 4 E
AG I
Roy
Induction by:
u.v. ( 30 sec.,
I0 cm. distance)
Actinomycin-C
(IO mcg./ml.)
H $\mathrm{O}_{2}$ (I0\%)

Parent strain

Small, wrinkled or rough

Slight, increases with age of culture

Long granulated rods, $95 \%$ acid fast

$24 \mathrm{hr}$ at $37^{\circ}$
$48 \mathrm{hr}$ at $22^{\circ}$
+++
-
++
++
++
\pm
++
+
+
++
+
+
++

Sensitive

Resistant

Resistant

-
+
+

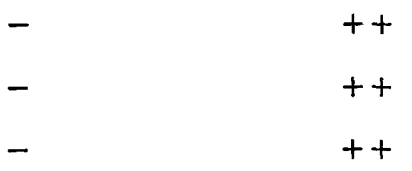

-

++
++
++
-
++
+
-
\pm
-
-
Lysogenic derivative

Large, smooth, nippled

Slight, appears immediately

Long and short (coccoid) bacilli. Loss of cytoplasma which retains less dye. Large acid fast granules (heavy 'beading') 4-5 days at $37^{\circ}$ 9 days at $22^{\circ}$

Sensitive

Resistant

Resistant

-
-
+
++
++
++

(Roy) and of M. lacticola (Roy) showed earlier and more pronounced pigmentation than did the respective parent strains. In contrast, strain 1302 (Roy) produced two kinds of colonies: one, pigmented as the parent strain; the other, nonpigmented and umbilicated. The above observations are summarized in Table 3. 
The time of appearance of lysogenic derivatives was studied with fast- and slowgrowing bacteria. The smooth colonies of 607 (Roy) appeared first in cultures plated $24 \mathrm{hr}$ after infection. They required nine days to attain maturity in these cultures. The slow-growing 1302 (Roy) colonies appeared Io days after phage infection. Their lag period was 5-6 days. After repeated subcultures, the lag periods were reduced considerably.

Comparison between strain 607 and its lysogenic derivative is summarized in Table 4. In addition to differences in colony morphology (Pl. I, fig. I), the microscopic aspect of the bacilli (Pl. I, fig. 2) and the changes in phagolytic pattern with resistance to phage Roy as well as to phage AG I, there was a loss of enzymic activity associated with lysogenization. These bacilli were not able to utilize paraffin as their sole carbon source. Their catalase reaction and nitrate reduction tests were negative; ammonium ferrocitrate was no longer transformed. The acetamidase reaction which already was weak in the parent strain, had completely disappeared. There was no change in the sensitivity to antimycobacterial drugs.

The lysogenic derivative of strain 1515 (Pl. I, fig. 3) showed almost the same changes as strain 607 (Roy): it produced smooth nippled colonies, it had acquired resistance to phagolysis by phage Roy and phage AG I and it had lost its nitrate reductase activity. Strain I5I8 (Roy) also showed resistance to phagolysis by phage Roy and phage AG I. In this case the lysogenic conversion was associated with the loss of nicotinamidase and urease activity. The non-pigmented umbilicated colonies of strain 1302 (Roy) (Pl. I, fig. 4) showed loss of their nicotinamidase, succinamidase and their nitrate reductase activity. Both the parent strain and the lysogenic variant are resistant to phagolysis. No changes in enzymic activity could be detected between the lysogenic strains of 1512, Mycobacterium phlei, $M$. stercoides, $M$. lacticola or the pigmented strain of 1302 .

\section{DISCUSSION}

Variations and 'adaptations' of mycobacteriophages have been reported many times. Among thirty or more phages isolated, in Redmond's laboratories only one had been free of some variations (Redmond, I963). Russell, Jann \& Froman (1963) have shown alterations in host range between lytic phages and their temperate derivatives. These authors suggested that 'some of these alterations may be due to host-controlled modification, while others may be due to the activation of a previously undetected prophage'. The latter mechanism, or recombination, was suggested by observations recently reported on the lytic phenomena of phage Leo propagated on ATCC 607 or on strain $\mathbf{H}_{37} \mathrm{RV}$ as alternate hosts (Mankiewicz \& Redmond, I968).

Failure to demonstrate a prophage in the bacterial parent cells may be due to lack of appropriate techniques or indicator strains, or to the particular state of the prophage. The prophage may be defective or inhibited in the expression of its genes by the action of a repressor present in the bacterial chromosome. Superinfection with phage Roy and recombination, however, may have caused the prophage to become detached from the chromosome.

Phage Roy does not lyse or lysogenize strains of Mycobacterium tuberculosis or representative strains from atypical mycobacteria group III. Only one of four strains of photochromogenic mycobacteria was susceptible to lysogenization by this phage. Strain 1518 has recently been isolated in our laboratories and has been classified as a 
photochromogen on the basis of photo-induced pigmentation, growth rate, amidase activity, phage-typing and the other above-mentioned tests. Scotochromogenic bacteria, $M$. fortuitum and fast-growing saprophytic mycobacteria appeared to be most susceptible to phage Roy infection.

In their recent study of the conversion of colony morphology and certain enzyme systems of ATCC607 lysogenized with phage D 29 or B4, Jones \& White (I968) described the appearance of phage-resistant smooth colonies which were lysogenic. Their morphology appears to be quite similar to the colonies of 607 (Roy) and of I5I5 (Roy). In contrast to the observations of these authors, strains lysogenized by phage Roy did not revert to the non-lysogenic state nor did they form rough colonies following subculture in media preventing phage readsorption. The high frequency of the loss of the lysogenic state together with the reversion to the formation of $R$ colonies by the 'cured' strain of 607 suggested to Jones and White that the genetic material that controls these changes is found in the extrachromosomal plasmids. This does not seem to apply to 607 (Roy) where the described changes were hereditary and permanent.

Jones \& White (1968) reported that the smooth colonies of 607 (D 29 or B 4) showed increased nitrate reductase activity and a higher degree of hydrolysis of Tween 80 . In this study the lysogenic derivative 607 (Roy) showed a reduction in the activity of several enzyme systems including that of the nitrate reductase.

These differences-provided they are not due to technical reasons-may indicate which of the bacterial enzyme systems affected by lysogenization are phage controlled and which are host determined. Similarities in colony conversion suggest that they are independent of the specific phage, while the different effects on the enzymic activity may be determined by the converting or recombinant phage. Further studies along these lines may help to clarify the process of lysogenization and the nature of certain mycobacterial variants.

Thanks are due to Dr V. Pavilanis and the staff of the Department of Virology, Université de Montréal, who performed the electronmicroscopic studies used in this project.

This project was supported in part by grants from the Medical Research Council of Canada and the Muskoka Hospital Memorial Research Fund.

\section{REFERENCES}

BoENICKE, R. (1958). Classification des mycobactéries à l'aide des épreuves chimiques. Bull. Un. int. Tuberc. 28, 162.

Jones, W. \& WhITE, A. (1968). Lysogeny in mycobacteria. I. Conversion of colony morphology, nitrate reductase activity, and Tween hydrolysis of Mycobacterium sp. ATcc607 associated with lysogeny. Can. J. Microbiol. r4, 551.

JUHASZ, S. E. \& BOENICKE, R. (1966). Reciprocal genetic changes in mycobacterial host-virus systems: effect of lysogeny on the phage. Nature, Lond. 210 , 186.

KonNo, K. (1957). The metabolism of nicotinic acid in mycobacteria: A method for differentiating tubercle bacilli of human origin from other mycobacteria. Am. Rev. Tuberc. 75, 529.

MANKIEWICZ, E. (196r). Morphological and biological properties of a strain of chromogenic acid-fast bacteria and of its phage-immune variant. J. gen. Microbiol. 24, 63 .

MANKmewicz, E. \& REDMOND, W. B. (I968). Lytic phenomena of phage Leo isolated from a sarcoid lesion. Am. Rev. resp. Dis. 98, $4 \mathrm{I}$.

27

G. Microb. 55 
REDMOND, W. B. (1963). Bacteriophages of the mycobacteria. A review. Adv. Tuberc. Res. 12, 19I. REDMOND, W. B. \& WARD, D. M. (1966). Media and methods for phage-typing mycobacteria. Bull. Wld. Hith Org. 35, 563 .

Russell, R. L., JANN, G. J. \& Froman, S. (1963). Lysogeny in mycobacteria. II. Some phage-host relationships of lysogenic mycobacteria. Am. Rev. resp. Dis. 88, 528.

TIRUNARANAY, M. \& FisChER, W. (1957). Relationship of isoniazid to the metabolism of mycobacteria. Am. Rev. Tuberc. 75, 62.

Tison, F., Taceutt, A. \& Devulder, B. (1964). Un test simple d'étude des mycobactéries: La transformation du citrate de fer ammoniacal. Annls Inst. Pasteur, Paris 106, 797.

VANDRA, E. \& TAKATs, A. (1965). Induced lysogenesis of mycobacteria. Acta microbiol. hung. 12, 29.

White, A., Foster, F. \& LYON, L. (I962). Alteration of colony morphology of mycobacteria associated with lysogeny. J. Bact. 84, 815 .

\section{EXPLANATION OF PLATE}

Fig. I. Conversion from rough to smooth nippled colonies of strain 607 and its lysogenic derivative 607 (Roy). (a) Wrinkled dry colonies; strain 607. (b) Smooth nippled colonies after lysogenization; strain 607 (Roy).

Fig. 2. Microscopic aspect of 607 (Roy) showing large granules and loss of acid fastness of cytoplasma. ( $\times$ 1400).

Fig. 3. Conversion from rough to smooth nippled colonies of strain 1515 and 1515 (Roy). (a) Rough dry colonies; strain 1515. (b) Smooth nippled colonies after lysogenization; strain 1515 (Roy).

Fig. 4. Dissociated into smooth pigmented and nonpigmented umbilicated colonies of strain 1302 and 1302 (Roy). (a) Round regular colonies; strain 1302. (b) After lysogenization, two types of colonies distinct from those of the parent strain: smooth pigmented and umbilicated non-pigmented colonies; strain I302 (Roy). 

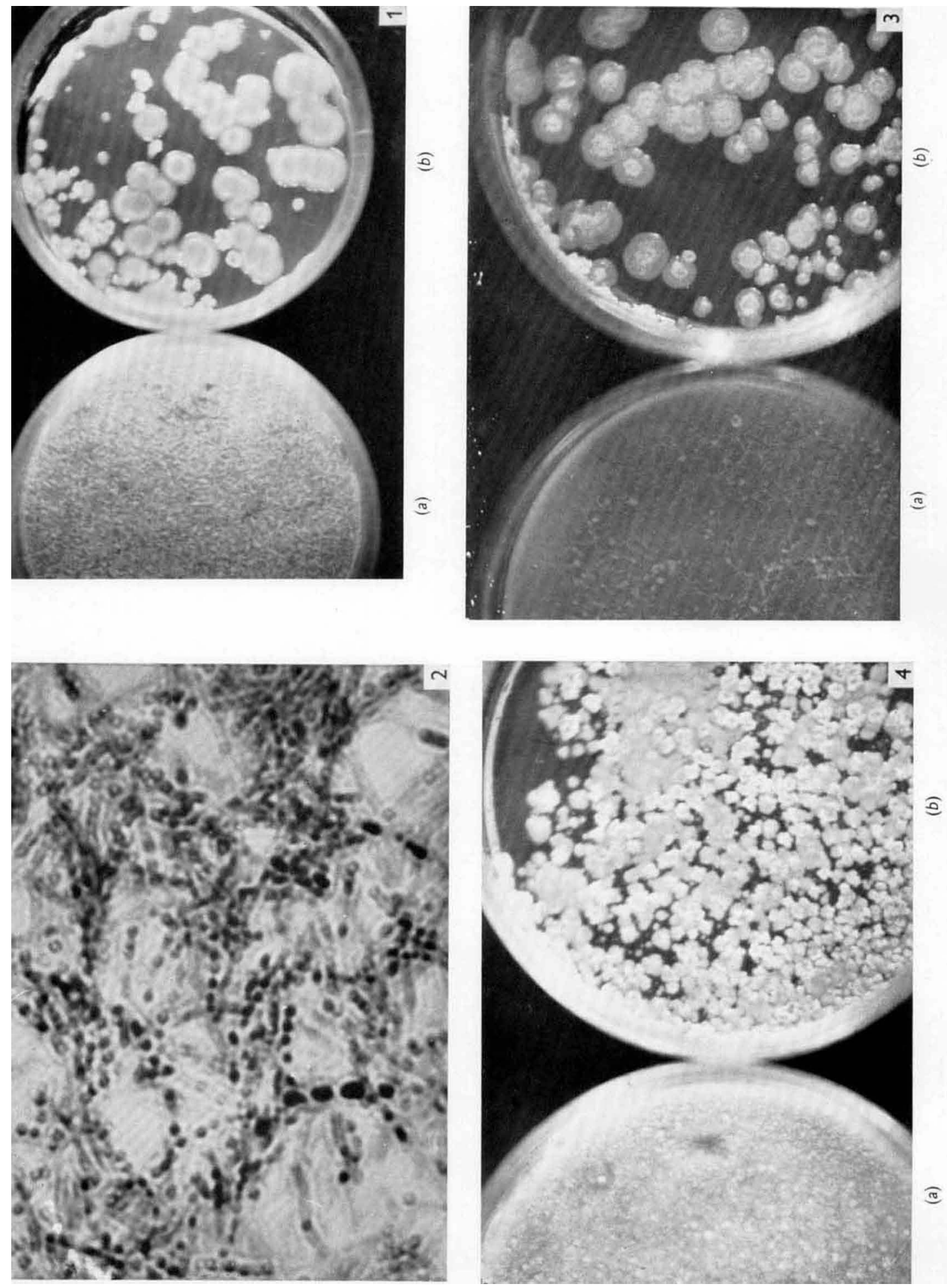

E. MANKIEWICZ AND OTHERS

(Facing p. 416) 